Oct 2016, Volume 1, Issue 3 (71-73)

\title{
Acute Compartment Syndrome of the Right Lower Extremity Following Autologous Blood Transfusion: A Case Report
}

\author{
Feridoun Sabzi ${ }^{1}$, Aghigh Heidari ${ }^{2}$, Fataneh Ghasemi ${ }^{3}$, Abbas
} Ahmadi ${ }^{4, *}$

\author{
${ }^{1} \mathrm{MD}$, Professor of Cardiovascular Surgery, Imam Ali Hospital Cardiovascular \\ Center, Kermanshah University of Medical Sciences, Kermanshah, Iran \\ ${ }^{2} \mathrm{MD}$, Assistant Professor of Cardiac and Anesthesiology, Imam Ali Hospital \\ Cardiovascular Center, Kermanshah University of Medical Sciences, Kermanshah, \\ Iran \\ ${ }^{3} \mathrm{MA}$, Anesthesia Technician, Imam Ali Hospital Cardiovascular Center, \\ Kermanshah University of Medical Sciences, Kermanshah, Iran \\ ${ }^{4} \mathrm{MD}$, Anesthesia Resident, Imam Ali Hospital Cardiovascular Center, Kermanshah \\ University of Medical Sciences, Kermanshah, Iran \\ * Corresponding author: Abbas Ahmadi, MD, Anesthesia Resident, Imam Ali Hos- \\ pital Cardiovascular Cente, Kermanshah University of Medical Sciences, Shahid Be- \\ heshti Boulevard, Kermanshah, Iran. Tel: +98-8338370252, Fax: +98-8338370252, \\ E-mail:abbasahmadi27i@yahoo.com
}

DOI: $10.21859 / \mathrm{ijcp}-010302$

Submited: 08.05.2016

Accepted: 09.07.2016

Keywords:

Compartment Syndromes

Blood Transfusion, Autologous

Cardiac Surgery

(C) 2016. International Journal of

Cardiovascular Practice.

\begin{abstract}
Compartment syndrome (CS) is an extremely rare complication during cardiac surgery and rare case reports have been linked to coronary artery bypass surgery.

We report one case of right lower extremity compartment syndrome (CS) following inadvertent blood transfusion through a catheter which was inserted into a vein in the related extremity. Forceful pushing of blood through a delicate vein led to rupture of the vein wall and subsequent extravasation of blood into the perivascular tissue as into an intra-compartment portion of the lower extremity.

Late detection of this complication led to compartement syndrome. The patient underwent emergency fasciotomy and concomitant removal of intra compartment and subcutaneous blood and fluids. After fasciotomy, the normal color of skin and pulse were recovered.
\end{abstract}

\section{INTRODUCTION}

Compartment syndrome (CS) is an extremely rare complication during cardiac surgery and rare case reports have been linked to coronary artery bypass surgery [1]. During harvesting of saphenous vein, some branches may be ruptured and then hidden in the subfascial space. These branches may bleed into the compartment space and lead to CS. Also, severe venous bleeding may be associated with subcutaneous hematoma which compresses the leg compartment [1]. Mild ischemia in this closet space is associated with edema and through a vicious cycle leads to CS. In these subjects, the first signs of compartment may be an excruciating extremity pain. However, if the case has not had a fast tract extubation, the golden time is over and amputation and neuromuscular deformity are devastating sequelae [2]. In rare cases, CS has been reported after anticoagulation therapy. In these cases, prescripting of anticoagulant druges to a patient with a congenital coagulation disorder was associated with bleeding in many sites such as extremity compartments [3]. Regardless of the etiology, if CS remains untreated, increased intracompartement pressure and devastating neuromuscular necrosis will result in sequela. We report a patient with CS of the right lower extremity following autologous blood transfusion after primary Bentall operation. This case, to our knowledge, was the first case of CS of the right lower extremity caused by a blood transfusion.

\section{CASE PRESENTATION}

A 55-year-old Iranian woman referred to our center with a history of dyspnea. Clinical and echocardiography evaluation showed severe and huge dilatation of ascending aorta and severe aortic insuficiency. She was scheduled for classic Bentall operation. In her medical history, no abnormal tendency to bleeding or other congenital or known hemophilia disorders were found. Her body mass index was $24 \mathrm{~kg} / \mathrm{m}^{2}$ and due to delicate vein wall and extensive subcutaneous fat, access to peripheral vein was difficult; however, a right central venous pressure (CVP) line and lower right extremity saphenous vein line were prepared for further transfusion. After midlinsternotomy, cardiopulmonary bypass was instituted and with moderate hypothermia, the ascending aorta just below the right subclavian vein was cross clamped and direct cardio- 
plegin infusion was performed in both coronary ostium. With cardioplegin arrest, the aneurismal segment of the ascending aorta resected and with separation of both coronary artery bottoms, the classic Bentall operation was performed. Just after opening the aortic cross clamp, venous bleeding from aortic root was detected. Due to massive bleeding from venous network around the right coronary buttom and a hemoglobin drop to $7.5 \mathrm{~g} / \mathrm{dL}$, blood transfusion with manual syrange pump from both peripheral lines was started by the nurses and the aortic root was packed with surgical sponges. Approximately 30 minutes following packing of the aortic root and blood transfusion, the patient was still hypotensive. At this time, anesthesiologist detected a deformity and rigidity in patient's right leg. It was deformed by 70 degrees flexion, was severely edematous from $10 \mathrm{~cm}$ upper the knee to the finger tips. Acral part of the fingers had cyanosis and multiple blisters were detected in the skin and pulse was not detected in the tibia or ulnar arteries. On palpation, both the volar and dorsal aspects of the leg were severely firm and rigid. The distal pulse was not detected by Doppler ultrasound. At that time, the subject had received $700 \mathrm{~mL}$ of packed blood cell and 2000 $\mathrm{mL}$ normal saline through a 16-gauge venous catheter inserted preoperatively in saphenous vein in the right lower leg. Retrospectely evaluating the situation, we found that the nurse sensed a resistance when she infused the packed cells through the intravenous catheter which was initially placed in the saphenous vein, but she thought that a mechanical problem in syringes was causing this resistance. It seems that the whole transfusion and normal saline were inadvertently infused into the extra venous interstitial tissue. CS was confirmed and extended fasciotomy was performed. Approximately $700 \mathrm{~mL}$ of hematoma was removed upon opening of the subcutaneous tissue and release of the superficial fascia, which was suspicious as the cause of CS. The deep fascia was noted to be tight and was released, and the dorsal compartment was released through a small window in the interosseous membrane. The fasciotomy incision was approximated using separate proline sutures. Immediately after fasciotomy, the pulse returned to distal arteries, cyanosis was relived and muscle consistency became soft and normal. Examination in the post-extubation period showed no pain with normal passive range of motion of fingers; however, she had paresthesias of medial aspect of the leg (Fig 1). At the $15^{\text {th }}$ day of the postoperative period, she had a residual strength deficit. The patient was discharged from the hospital on the $20^{\text {th }}$ day postoperative. The patient was examined one, two and three weeks after discharge from the hospital. The incisional leg wound was healed at that time without the presence of erythema or drainage. However, the case continued to have a residual paresthesia on medial aspect of the right thumb at that time.

\section{DISCUSSION}

In cardiac surgery cases after general anesthesia, symptoms such as pain have not been found in CS and the first sign may be resistance in syringes used for pushing blood in these emerging patients. Unfortunately, if this issue is linked to mechanical or structural problems of syringes, the anesthesiologist and nurse may be heedless of this major complication and continue the forceful transfusion. With rupture of the vein wall and continuation of transfusion, especially in a full anticoagulation state, more subcuataneous and intra-compartment space get opened for further inadvertent transfusion. In this stage, the resistance to infusion reduces and through a vicious cycle, a copious amount of fluids may be infused into subcutaneous and intra-compartment of the extremity. In this stage in our study, the skin blister with an abnormal flexion position in the related extremity appeared. Due to coverage of extremity in non-coronary cases, the flexion of extremities may be a late and single sign of this rare complication [4]. Early detection of CS of any etiology is important because it can lead to irreversible injury if remained untreated. Calf is the rarest site of CS amongst the four extremities. Pain is the most important symptom in awake cases; also, pain with passive extension of fingers has been exhibited to be the most sensitive sign of CS. Detection of a palpable tibial pulse is equivocal in the diagnosis of CS in complicated cases [5]. Functional impairment of muscles was observed from 120 minutes to 240 minutes of ischemia; but in nerve tissue, abnormal function has been seen after $30 \mathrm{~min}$ utes. Matsen et al. exhibited that if fasciotomy is delayed for 720 minutes or more, only $7 \%$ could have a normal neuromuscular function vs. $70 \%$ who will have on time fasciotomy within 720 minutes [6]. The etiology of CS in our case was unique; our hypothesis is that there was a technical problem in catheter insertion that caused a malfunction in catheter. However, misplacement of the catheter into extra venous tissue leads to extravasation of blood to the soft tissue. The catheter could have been placed through the vein and into the subcutaneous and superficial fascial compartment. It is noteworthy that copious amount of hematoma $(700 \mathrm{~mL})$ was removed after the release of the superficial fascia. In careful literature search, we found no previous reported studies that described calf CS from such a small amount of extravasated fluid. However, intravenous catheter is a common cause of extravasation of fluids in forearm area, but has rarely led to CS, because the forearm veins have an extra-compartmental position. Kagel et al. showed one forearm CS of 67 intravenous catheter misplacement during a 3-year period [7]. Our case confirmed the importance of avoiding a placement of catheter in a lower extremity vein in cardiac surgery cases where lower extremity is covered; however, intra-compartmental pressures exam is a good option in a cardiac case where the risk of CS is high. Another risk factor for occurrence of CS in our case was prolonged postoperative hypotension. Hypotension is believed to be a predisposing factor to CS [8]. This case highlighted the importance of possible intravenous catheter misplacement or rupture during blood infusion. This case also showed that despite the presence of an extensive network of vein that connects the superficial veins to intra-compartment parts, extravasation of large amounts of fluid into the superficial tissue with compression of lower compartment can potentially lead to CS.

\section{CONFLICTS OF INTEREST}

There is no conflict of interest for the present study.

\section{ACKNOWLEDGMENTS}

There is no acknowledgment for the present study.

\section{REFERENCES}

1. Al-Sarraf N, Al-Shammari F, Vislocky I, Malek L. Lower limb compartment syndrome following coronary artery bypass surgery: a rare entity. Gen Thorac Cardiovasc Surg. 2010;58(3):131-3. DOI: 
10.1007/s11748-009-0488-z PMID: 20349302

2. Te Kolste HJ, Balm R, de Mol B. Acute Compartment Syndrome of the Lower Leg after Coronary Artery Bypass Grafting: A Silent but Dangerous Complication. Thorac Cardiovasc Surg. 2015;63(4):3006. DOI: $10.1055 / \mathrm{s}-0034-1395443$ PMID: 25602845

3. Rodriguez-Merchan EC. Acute compartment syndrome in haemophilia. Blood Coagul Fibrinolysis. 2013;24(7):677-82. DOI: 10.1097/MBC.0b013e3283631e1a PMID: 24064900

4. Mabvuure NT, Malahias M, Hindocha S, Khan W, Juma A Acute compartment syndrome of the limbs: current concepts and management. Open Orthop J. 2012;6:535-43. DOI: 10.2174/1874325001206010535 PMID: 23248724
5. Bowyer MW. Compartment Syndrome of the Extremities. Penetrating Trauma: Springer; 2012. p. 427-36.

6. Matsen FA, 3rd, Winquist RA, Krugmire RB, Jr. Diagnosis and management of compartmental syndromes. J Bone Joint Surg Am. 1980;62(2):286-91. PMID: 7358759

7. Kagel EM, Rayan GM. Intravenous Catheter Complications in the Hand and Forearm. J Trauma Injury Infection Critical Care. 2004;56(1):123-7. DOI: 10.1097/01.ta.0000058126.72962.74 Donaldson J, Haddad B, Khan WS. The pathophysiology, diagnosis and current management of acute compartment syndrome. Open Orthop J. 2014;8:185-93. DOI: 10.2174/1874325001408010185 PMID: 25067973 